\title{
Direct imaging of repulsive and attractive colloidal glasses
}

\section{Citation}

Kaufman, Laura J., and David A. Weitz. 2006. "Direct Imaging of Repulsive and Attractive Colloidal Glasses." The Journal of Chemical Physics125 (7): 074716. https:// doi.org/10.1063/1.2227386.

\section{Permanent link}

http://nrs.harvard.edu/urn-3:HUL.InstRepos:41511262

\section{Terms of Use}

This article was downloaded from Harvard University's DASH repository, and is made available under the terms and conditions applicable to Other Posted Material, as set forth at http:// nrs.harvard.edu/urn-3:HUL.InstRepos:dash.current.terms-of-use\#LAA

\section{Share Your Story}

The Harvard community has made this article openly available.

Please share how this access benefits you. Submit a story.

Accessibility 


\title{
Direct imaging of repulsive and attractive colloidal glasses
}

\author{
Laura J. Kaufman ${ }^{\text {a) }}$ \\ Department of Chemistry, Columbia University, New York, New York 10027 \\ David A. Weitz \\ Division of Engineering and Applied Science, Harvard University, Cambridge, Massachusetts 02138 \\ and Department of Physics, Harvard University, Cambridge, Massachusetts 02138
}

(Received 3 March 2006; accepted 20 June 2006; published online 21 August 2006)

\begin{abstract}
Coherent anti-Stokes Raman scattering microscopy is performed on glassy systems of poly(methylmethacrylate) colloidal particles in density- and refractive-index-matched solvents. Samples are prepared with varying amounts of linear polystyrene, which induces a depletion driven attraction between the nearly hard-sphere particles. Images collected over several hours confirm the existence of a reentrant glass transition. The images also reveal that the dynamics of repulsive and attractive glasses are qualitatively different. Colloidal particles in repulsive glasses exhibit cage rattling and escape, while those in attractive glasses are nearly static while caged but exhibit large displacements upon (infrequent) cage escape. () 2006 American Institute of Physics.
\end{abstract}

[DOI: $10.1063 / 1.2227386]$

\section{INTRODUCTION}

It has been well established via experiment ${ }^{1-4}$ and simulation ${ }^{5-9}$ that particles with hard-sphere or nearly hard-sphere interactions undergo entropically driven phase transitions as a function of particle volume fraction $\phi$. Experiments on three-dimensional (3D) systems of nearly hardsphere colloidal particles composed of poly(methylmethacrylate) (PMMA) spheres with short poly(12-hydroxystearic acid) (PHSA) chains on the surface have shown the variety of accessible phases. At $\phi<0.495$, monodisperse colloidal particles diffuse freely, for $0.495<\phi<0.545$ there is liquidcrystal coexistence, and at $\phi>0.545$ colloidal particles exist in a crystalline state. ${ }^{1,4,5}$ Colloids can also be induced into a (nonequilibrium) glassy state by using centrifugation to rapidly increase the volume fraction from $\phi<0.495$ to $\phi$ $\sim 0.58-0.64$ (Refs. 1,2 , and 10) [with $\phi=0.64$ traditionally defining random close packing (RCP) for monodisperse hard spheres ${ }^{8,11,12}$. Dynamic light scattering (DLS) experiments show that these colloidal glasses do not relax completely on the longest time scales probed (as long as $10^{4}-10^{5} \mathrm{~s}$ ). ${ }^{2,10,13,14}$

Colloidal glasses display much of the same phenomenology as molecular glassy systems. From a macroscopic point of view, colloidal glasses appear to be solid, ${ }^{15}$ but microscopically they lack long range order, as is true of molecular glasses. In addition, much as dynamics in molecular systems slow precipitously as the glass transition temperature is approached, so too do the dynamics of colloidal systems slow as the glass transition density is approached. However, in colloidal glasses, $\phi$ is the thermodynamic control parameter rather than temperature $T$, as is the case in molecular glasses. In molecular systems, slow dynamics are accompanied by nonexponential decays of commonly measured observables, ${ }^{16}$ such as the dielectric permitivity measured by dielectric spectroscopy and the rotational correlation func-

${ }^{a)}$ Electronic mail: kaufman@chem.columbia.edu tions measured by single molecule spectroscopy. ${ }^{17}$ It is now fairly well accepted that both heterogeneous dynamics and cooperative rearrangements as first discussed by Adam and Gibbs $^{18}$ are intimately related to these nonexponential relaxations. Whether dynamic heterogeneity and cooperative rearrangement are also important in the slow dynamics of colloidal glasses has recently been explored at an unprecedented level of detail through confocal fluorescence microscopy studies that measure the trajectories of many individual particles within the systems for long times. ${ }^{19-23}$ These experiments have confirmed that the dynamics in glassy colloidal systems are heterogeneous and have suggested that the rearrangements they undergo are cooperative.

While hard-sphere colloidal systems exhibit a wide range of interesting behavior, colloidal systems with only slightly more complicated interaction potentials have been shown to exhibit an even broader array of phases and dynamics. Studies on colloidal particles with strong charge repulsion have uncovered low volume fraction crystals and glasses, ${ }^{24}$ a subset of which were recently examined with confocal fluorescence microscopy. ${ }^{25}$ Studies have also shown that colloidal systems with short range attractions exhibit interesting phase behavior and dynamics. ${ }^{26-31}$ For these systems, the depth of the attractive interaction $U$ becomes an additional important control parameter; since it is typically normalized by thermal energy, $U / k_{B} T$, it mimics the role of inverse temperature in analogy to molecular glasses.

One of the most intriguing examples of interesting phase behavior in attractive colloidal systems was first predicted by calculations using mode coupling theory (MCT) that suggested that colloids with short range attractions could experience a reentrant glass transition: ${ }^{32,33}$ this describes phase behavior of a high density system that transforms from glassy to liquidlike and back to glassy with increasing depth of the attractive potential. Subsequent simulations ${ }^{34-36}$ and DLS experiments ${ }^{29-31}$ confirmed the presence of both the 


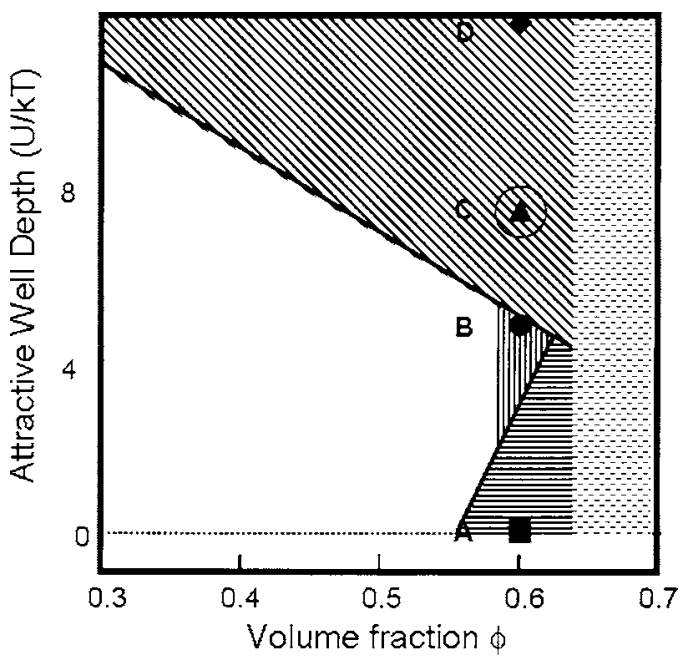

FIG. 1. Possible reentrant phase diagram with repulsive and attractive glass lines adapted from Pham et al. The area with horizontal stripes represents the repulsive glass and the area with diagonal stripes represents the attractive glass (and gel) region. Area with vertical stripes represents a possible reentrant region. Details are discussed in the Experiment and Results and Discussion sections. Samples examined in these experiments are represented by points $\mathrm{A}-\mathrm{D}$. Beyond $\phi=0.62$, there may be a region in which there is a glass-glass phase transition and a region in which the two glasses are indistinguishable.

reentrant glass transition and two types of glasses. These glasses, termed the repulsive and attractive glasses, have dynamics dictated by the repulsive and attractive portions of the potential, respectively. Figure 1 presents a phase diagram adapted in part from Pham et al. showing the qualitative features expected in high volume fraction colloidal systems with short range attractions. ${ }^{29}$ The line at zero polymer concentration represents the phase behavior for hard-sphere colloids, which experience a glass transition at $\phi \sim 0.58$. In Fig. 1 , the area with horizontal stripes represents the repulsive glass region and the area with diagonal stripes represents the attractive glass region. The area between these regions, shaded with vertical lines, is termed the reentrant region. MCT calculations have shown that at higher volume fractions, the lines defining the repulsive and attractive glass transitions will meet, and beyond that point there may be a glass-glass transition. At even higher volume fractions, the attractive glass line extends to a singularity known as the A3 point beyond which attractive and repulsive glasses will be indistinguishable.

Experimentally, attraction between colloidal particles is induced by adding small amounts of nonadsorbing linear polymer to the system. There is high entropic cost for polymer coils to reside between two particles that are close together compared to the radius of gyration $\left(r_{g}\right)$ of the polymer. Thus, the polymer is unlikely to reside between such sets of colloids, which leads to an increased osmotic pressure on and a depletion induced attraction between such colloidal particles. In DLS experiments on attractive glasses, the intermediate scattering function, $f(q, \tau)$, which reports on the relaxation of the system, is measured. In the experiments of Pham et al., a glass is defined as a sample with $\phi>0.565$ and an $f(q, \tau)$ that does not decay to zero on the time scale of the experiment $\left(\sim 10^{4} \mathrm{~s}\right) .{ }^{29,31}$ High density colloidal systems with no polymer and with small amounts of linear polymer have similar $f(q, \tau)$, each displaying a decay into a distinct plateau followed by a second decay at long times. The plateau is typically associated with caged behavior, and the subsequent decay is typically associated with cage escape and structural relaxation. For systems with somewhat more polymer, $f(q, \tau)$ is found to decay to zero in $<10^{4} \mathrm{~s}$. Thus, the samples with intermediate polymer concentration behave like liquids in terms of their relaxation even though samples at the same volume fraction with less polymer do not. If yet more polymer is introduced, the system reverts to glassy behavior, though the shape and aging behavior of $f(q, \tau)$ differ significantly from that of glasses with no or small amounts of polymer. For colloidal systems with strong attractions $f(q, \tau)$ exhibits a very slow decay and no clear plateau. The DLS data on these systems, thus, show that a reentrant glass transition and two distinct types of glasses, repulsive and attractive, exist in high density colloidal systems with short range attractions. However, the relationship between the motion and the attractions in these systems is best measured with direct imaging of the particles, and this has not, as yet, been reported.

In this paper, we present the first microscopic visualization of colloidal glasses with short range attractions, which allows new insight into the dynamics of these systems. We perform the first experiments that examine systems in the repulsive glass, reentrant, and attractive glass regions with microscopy. We use coherent anti-Stokes Raman scattering (CARS) microscopy to confirm the presence of the reentrant glass transition and examine the microscopic differences between the dynamics in the repulsive and attractive glasses. We show that the dynamics of the attractive glass are qualitatively different than those of the repulsive glass. While the repulsive glass dynamics are well characterized by cage rattling and escape, attractive glass dynamics are characterized by very little motion within tight cages and infrequent, saltatory motion that leads to fast, cooperative rearrangements of particles.

\section{EXPERIMENT}

Sterically stabilized colloids with radius of $1.2 \mu \mathrm{m}$ and polydispersity of $6 \%$ (as measured by scanning electron microscopy) are received in dodecane. The colloids are composed of PMMA and have short, stiff PHSA molecules grafted onto the surface, which prevents aggregation and leads to nearly hard-sphere interactions between the particles. Through repeated washings, samples are transferred first into decalin and then into density- and nearly refractiveindex-matched mixtures of decalin, cyclohexyl bromide (CXB), and tetrabutyl-ammonium chloride (TBAC). TBAC is added at $2 \mathrm{mM}$ concentration to screen any possible Coulombic interactions due to charges on the colloidal particles dispersed in the solvents used here. ${ }^{25}$ The particles are then placed in an oven at $80{ }^{\circ} \mathrm{C}$ for several hours. This heat-shock procedure allows the particles to swell in the solvents and ensures they do not swell further during the experiment. In our samples any swelling from this procedure is minimal, as the average particle radius measured by CARS microscopy 
remains $1.2 \mu \mathrm{m}$. Centrifugation is used to concentrate the particles to RCP. The RCP volume fraction for these particles is found via drying experiments: particles are weighed after centrifugation and removal of effluent, allowed to dry thoroughly, and weighed again. Drying experiments show that $\mathrm{RCP}$ in this sample is $66 \pm 1 \%$. This is higher than RCP for monodisperse spheres and is consistent with a polydispersity of $6 \%{ }^{8,37}$ The error on all volume fractions presented in this manuscript is set by the error in the measurement of RCP.

Samples are diluted from RCP to $\phi=0.60$ using a combination of effluent from the centrifugation and a separate solution containing $\mathrm{CXB}$, decalin, TBAC, and $10 \mathrm{mg} / \mathrm{ml}$ linear polystyrene (PS) with a molecular weight of $13.2 \times 10^{6} \mathrm{Da}$. This allows the preparation of the four samples detailed in this manuscript: sample A $(\phi=0.60$, $\mathrm{PS}=0 \mathrm{mg} / \mathrm{ml})$, sample B $(\phi=0.60, \quad \mathrm{PS}=0.22 \mathrm{mg} / \mathrm{ml})$, sample C $(\phi=0.60, \quad \mathrm{PS}=0.44 \mathrm{mg} / \mathrm{ml})$, and sample D $(\phi=0.60, \mathrm{PS}=0.88 \mathrm{mg} / \mathrm{ml})$. Additional samples with $0,0.22$, and $0.44 \mathrm{mg} / \mathrm{ml}$ PS are prepared at $\phi=0.58$, as is one sample at $10 \mathrm{mg} / \mathrm{ml}$ PS and $\phi=0.60$. Samples at their final compositions are tumbled for at least two days before being transferred via glass pipette to microscopy sample cells. In some samples, small magnetic stir bars are added to allow the samples to be mixed and hence reinitiated. No qualitative or quantitative differences have been found between samples initiated via the stir bar and those initiated by sample loading, though those without stir bars cannot be reproducibly reinitialized by tumbling or shaking of the small sample cells. After initiation, samples are allowed to rest for $2 \mathrm{~h}$ before CARS microscopy measurements begin.

CARS microscopy ${ }^{38-41}$ has been employed to study the structure and dynamics of these samples. Contrast in CARS microscopy is based on Raman active vibrations inherent to the sample that are excited and interrogated by two picosecond lasers. The frequencies of these lasers, the pump $\left(\omega_{p}\right)$ and Stokes $\left(\omega_{S}\right)$ frequencies, are set such that the difference frequency, $\omega_{p}-\omega_{S}$, is resonant with a vibration in the sample. The lasers excite a coherent oscillation of vibrations in the sample, which is interrogated by a probe pulse with the same frequency as the pump pulse. At each point in time a small focal volume $\left(\sim 0.4 \times 0.4 \times 1.2 \mu \mathrm{m}^{3}\right)$ is illuminated, and CARS signal is detected in the forward direction on a photomultiplier tube. The lasers are scanned over the sample, and an image is produced (Fig. 2). CARS microscopy does not require the incorporation of fluorophores into the system and thus circumvents two difficulties associated with fluorescence microscopy, potential perturbation to the system and long time bleaching. The absence of bleaching is especially advantageous in imaging colloidal glasses, for we wish to collect images continuously for long times. With CARS microscopy, the limiting factors in length of data collection are drift in the $z$ position of the microscope objective and drift in the time overlap of the pump and Stokes lasers.

Here, the pump and Stokes frequencies are set so that $\omega_{p}-\omega_{S}=2842 \mathrm{~cm}^{-1}$, a strongly Raman active transition in decalin. In the images presented here (see Fig. 2, for example) the contrast is inverted so that the particles, rather than the solvent, appear bright. The average laser powers measured before the microscope scan head and objective are

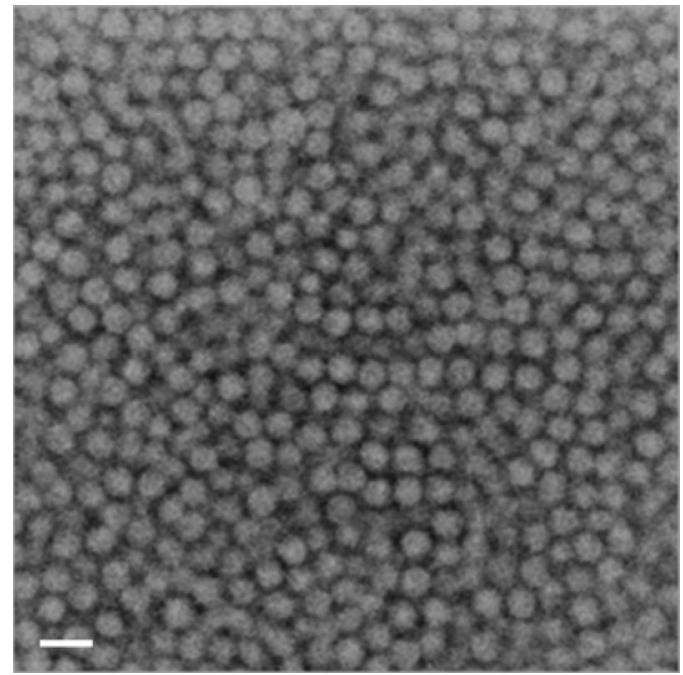

FIG. 2. Typical CARS image of PMMA colloids of diameter of $\sim 2.4 \mu \mathrm{m}$. Pump frequency $\omega_{p}$ is $14076 \mathrm{~cm}^{-1}$ and Stokes frequency $\omega_{S}$ is $11234 \mathrm{~cm}^{-1}$. The frequency difference, $2842 \mathrm{~cm}^{-1}$, is resonant with a strongly Raman active vibration in decalin. The image contrast is inverted to make the colloids (rather than the solvent) appear bright. Image collected in 3.9 s. Scale bar is $5 \mu \mathrm{m}$.

held to $\sim 20 \mathrm{~mW}\left(\omega_{p}\right)$ and $50 \mathrm{~mW}\left(\omega_{S}\right)$. Two-dimensional (2D) slices of $514 \times 514$ pixels within the $3 \mathrm{D}$ sample are collected continuously, with each image taking 3-4 s to acquire. All images are taken at least $40 \mu \mathrm{m}$ into the sample to avoid effects from the coverslip, including layering of the particles. Three-dimensional stacks of 2D images have also been collected, but the full 3D images are not analyzed in this manuscript due to the poorer resolution along the $z$ axis than in the $x-y$ plane.

The images of samples A-D are analyzed using particle tracking algorithms developed by Crocker and $\mathrm{Grier}^{42}$ and implemented in fluorescent confocal imaging of glassy colloidal systems. ${ }^{19,21,22,43,44}$ The volume fraction and polymer concentration of the samples interrogated were chosen so as to probe a variety of phases explored in the DLS studies of Pham et $a l . .^{29,31}$ The majority of the samples studied have $\phi \sim 0.60$, giving $f=\left(\phi_{\text {rep }} \sim \phi\right) / \phi_{\text {rep }}=0.09$, which is similar to that of the samples studied with DLS in Refs. 29 and 31. The systems explored in those references were composed of smaller colloidal particles and linear polymers than employed in this study. Therefore, to identify samples at the same positions on the phase diagram in both sets of experiments, we use size and concentration data given in those references to calculate potentials for a number of samples probed in those works. Then, in our system, polymer concentrations are chosen so as to reproduce those potentials.

To calculate the potentials we use the Asakura-Oosawa form $^{45-47}$ and calculate the potential as detailed by Ilett et $a{ }^{48}$ The potential is given by

$$
U= \begin{cases}\infty & \text { for } r \leqslant \sigma \\ -\Pi_{p} V_{\text {overlap }} & \text { for } \sigma<r \leqslant \sigma+2 r_{g} \\ 0 & \text { for } r>2 r_{g},\end{cases}
$$




$$
V_{\text {overlap }}=\frac{\pi}{6} \sigma^{3}(1+\xi)^{3}\left(1-\frac{3 r}{2 \sigma(1+\xi)}+\frac{1}{2}\left[\frac{r}{\sigma(1+\xi)}\right]^{3}\right),
$$

and

$$
\Pi_{p}=c_{p}^{\mathrm{free}} / k T,
$$

where $c_{p}^{\text {free }}=N_{p} / V_{\text {free. }}$ In these equations, $r$ is distance, $\sigma$ is the particle diameter, $r_{g}$ is the radius of gyration of the polymer, and $\xi$ is the ratio of radii of the polymer and particle $\left(\xi=r_{g} / a\right)$, which sets the range of the interaction. $V_{\text {overlap }}$ is the volume of overlapping depletion zones between two particles separated by a distance $r$. This quantity is determined by geometric considerations and assumes both the particles and coiled polymers are spherical. $\Pi_{p}$ is the osmotic pressure, which increases with increasing polymer concentration. $c_{p}^{\text {free }}$ is the concentration of polymer coils in the free volume and $N_{p}$ is the number of polymer coils in the sample. $V_{\text {free }}$ is the volume not occupied by either the colloidal particles or their associated depletion regions. $V_{\text {free }}$ is determined via scaled particle theory ${ }^{26,49,50}$ with

$$
\begin{aligned}
& V_{\text {free }}=\alpha V, \\
& \alpha=(1-\phi) \exp \left(-A \gamma-B \gamma^{2}-C \gamma^{3}\right),
\end{aligned}
$$

where $\gamma=\phi /(1-\phi), A=3 \xi+3 \xi^{2}+\xi^{3}, \quad B=9 \xi^{2} / 2+3 \xi^{3}$, and $C=3 \xi^{3}$.

While polymer concentration sets the strength of the attractive interaction, $\xi$ sets the range of the interaction. It has been suggested that the reentrant glass transition will only occur for short range attractive interactions, with $\xi<0.15 .^{33}$ Static light scattering (SLS) was employed to find the $r_{g}$ of the large linear polystyrene employed in this study at very low concentrations $\left(r_{g}(0)\right)$. SLS on the $13.2 \times 10^{6} \mathrm{Da}$ PS studies resulted in noisy data, so SLS was done on a variety of PS between $3 \times 10^{5}$ and $11.4 \times 10^{6} \mathrm{Da}$. In this molecular weight range, the data were well fit by the relation $r_{g}^{a}(0)=\left(M_{w}^{a} / M_{w}^{b}\right)^{0.56} r_{g}^{b}(0)$. The same relation was then used to find $r_{g}(0)$ for the $13.2 \times 10^{6} \mathrm{Da}$ PS. It is determined that $r_{g}(0)$ of the $13.2 \times 10^{6} \mathrm{Da}$ PS is $145.4 \mathrm{~nm}$ and $\xi=0.12$. This result is consistent with previous findings for polystyrene in similar solvents. ${ }^{51,52}$ The calculated radius of gyration gives an overlap concentration of $1.7 \mathrm{mg} / \mathrm{ml}$.

To calculate the attractive potentials in both our samples and the samples studied in Refs. 29 and 31, we must take into account that $r_{g}$, and consequently, $\xi, V_{\text {overlap}}$, and $\Pi_{p}$ are all concentration dependent. The radius of gyration is a function of concentration since the polymer coil is compressed as free volume decreases. This is accounted for using relations derived from Ohta-Oono conformation space renormalization theory. ${ }^{53}$

$$
\begin{aligned}
\frac{M_{w}}{R T} \frac{d \Pi_{p}}{d c}= & 1+\frac{1}{8}\left(9 \hat{X}-2+\frac{2 \ln (1+\hat{X})}{\hat{X}}\right) \\
& \times \exp \left(\frac{1}{4}\left(\frac{1}{\hat{X}}+\left(1-\frac{1}{\hat{X}^{2}}\right) \ln (1+\hat{X})\right)\right),
\end{aligned}
$$

with $\hat{X}=\frac{16}{9}\left(c_{p}^{\text {free }} / c^{*}\right)$. The overlap concentration $c^{*}$ is given by

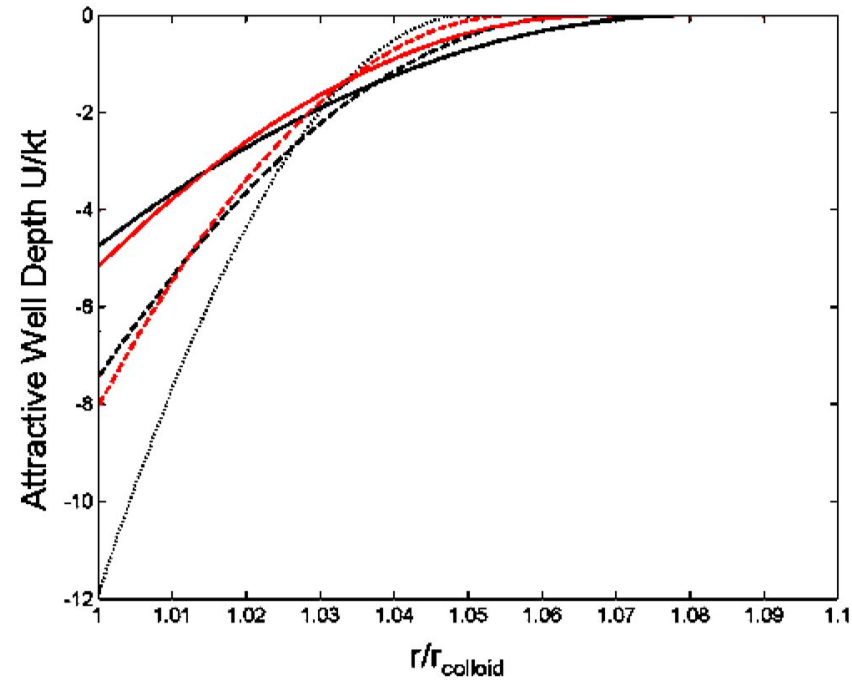

FIG. 3. Potentials for sample B $(0.22 \mathrm{mg} / \mathrm{ml}$ PS, dark solid line), sample C $(0.44 \mathrm{mg} / \mathrm{ml} \mathrm{PS}$, dark dashed line $)$, and sample D $(0.88 \mathrm{mg} / \mathrm{ml}$, dotted line $)$. Potentials calculated for samples B $(2.1 \mathrm{mg} / \mathrm{ml}$ PS, light [red] solid line) and $\mathrm{E}(4.4 \mathrm{mg} / \mathrm{ml}$, light [red] dashed line) in Ref. 29.

$c^{*} \approx M_{w} /\left[(4 \pi / 3) r_{g}^{3}(0) N_{A}\right]$, with $N_{A}$ Avogadro's number. Finally, $r_{g}(c)=r_{g}(0)\left(\sqrt{\left(M_{w} / R T\right)\left(d \Pi_{p} / d c\right)}\right)^{-1}$. From this expression, all other concentration dependent quantities can be calculated, though since $r_{g}$ and $\Pi_{p}$ depend on each other, determining these quantities is done in an iterative fashion. Potentials calculated for two samples used in Ref. 29, a repulsive glass and a reentrant sample, are shown in Fig. 3, together with potentials calculated for three samples used in this study. We note that while $\xi=0.12$ assuming $r_{g}(0)$ for our experiment, for the samples used, $\xi$ ranges from 0.08 for sample B to 0.05 for sample D, with $r_{g}(c)$ ranging from $98 \mathrm{~nm}$ for sample B to $61 \mathrm{~nm}$ for sample D.

\section{RESULTS AND DISCUSSION}

\section{Detailed phase diagram}

Figure 1 is a phase diagram adapted from Pham et al. ${ }^{29,31}$ Equation (1) was used to convert the total polymer concentration as reported in Refs. 29 and 31 to attractive well depth. The area identified as reentrant spans a small region in $\phi$ between 0.59 and 0.61 . In this region, Pham et al. found samples that crystallized over times as short as one day. Before crystallization these samples displayed ergodic, liquidlike behavior in DLS experiments. At the same polymer concentrations but for volume fractions above $\phi$ $=0.62$, samples with some characteristics of both attractive and repulsive glasses were identified by both visual inspection and DLS experiments. ${ }^{31}$ The logarithmic relaxation of $f(q, \tau)$ of these samples suggests that they are close to the $\mathrm{A} 3$ point predicted by MCT. ${ }^{33,34,54}$

The positions of the four samples to be detailed here have been superimposed on the phase diagram in Fig. 1. Because the polymer used in our study is much larger than that used in the DLS studies, the total polymer concentration employed here is lower than in those studies. However, the potentials are nearly identical between two of our samples and two studied by Pham et al. as was shown in Fig. 3. 
While Pham et al. identify a sample with a potential almost identical to that of our sample $\mathrm{C}$ as an attractive glass, we find indications that it is in the reentrant region or very close to the reentrant/attractive glass transition line. We circle the symbol representing sample $\mathrm{C}$ in Fig. 1 to indicate this inconsistency between the phase diagram adapted from Pham et al. and our results. As mentioned previously, samples at 0 , 0.22 , and $0.44 \mathrm{mg} / \mathrm{ml}$ PS but at $\phi=0.58$ were also prepared. These samples were used to investigate whether the crystallization/glass line moves to higher volume fractions as a function of polymer concentration. Both of the $\phi=0.58$ samples containing polymer were found to crystallize quickly, which is consistent with the results of Pham et al. and with the glass transition line moving to higher volume fractions. The dynamics of this crystallization, which occurred in the first $6 \mathrm{~h}$ following sample loading, will not be discussed in this manuscript. In all other samples measured, we do not observe macroscopic crystallization as defined by visual observation of opalescence in the samples loaded in microscopy sample cells for many weeks.

\section{Structure}

Before examining dynamics as assessed by both ensemble averaged quantities and visual observation, the samples are examined for differences in structure. As polymer is added to the system, the increasingly attractive interaction is expected to compact the cage of neighbors surrounding a particular particle. However, because the overall volume fraction is the same in all samples studied, compacted cages with higher local volume fraction must necessarily be accompanied by regions in the sample that are on average lower in volume fraction. These areas could manifest as voids in the sample or as subtle differences in the colloidal organization, with tighter cages on the nearest neighbor length scale, but looser organization on longer length scales. Another possibility is that the cages in samples with attractive interactions consist of more particles on average than do those in hard-sphere systems. Such organization could allow for higher local volume fraction in attractive cages than in hard-sphere cages while maintaining the same overall volume fraction in the sample. Visual inspection of the CARS images does not show any structural difference between samples A-D. However, samples with much higher polymer concentration are structurally different than samples A-D. In a sample with $10 \mathrm{mg} / \mathrm{ml}$ PS, which is well above the overlap concentration $c^{*}$, clumps of colloidal particles and voids are apparent. This sample is not included in any of the ensemble averaged dynamics data to be presented since the sample appears sufficiently spatially heterogeneous such that ensemble averaged quantities cannot provide much insight into its structure or dynamics.

To look for subtler differences in structure between samples $\mathrm{A}, \mathrm{B}, \mathrm{C}$, and $\mathrm{D}$, the radial distribution functions, $g(r)$, are presented in Fig. 4. Sample D $(0.88 \mathrm{mg} / \mathrm{ml}$ PS, dotted line) has a $g(r)$ with significantly more structure than do any of the other samples, indicating increased compaction. Though increased structure is often seen in samples with some crystallization, we find no macroscopic or micro-

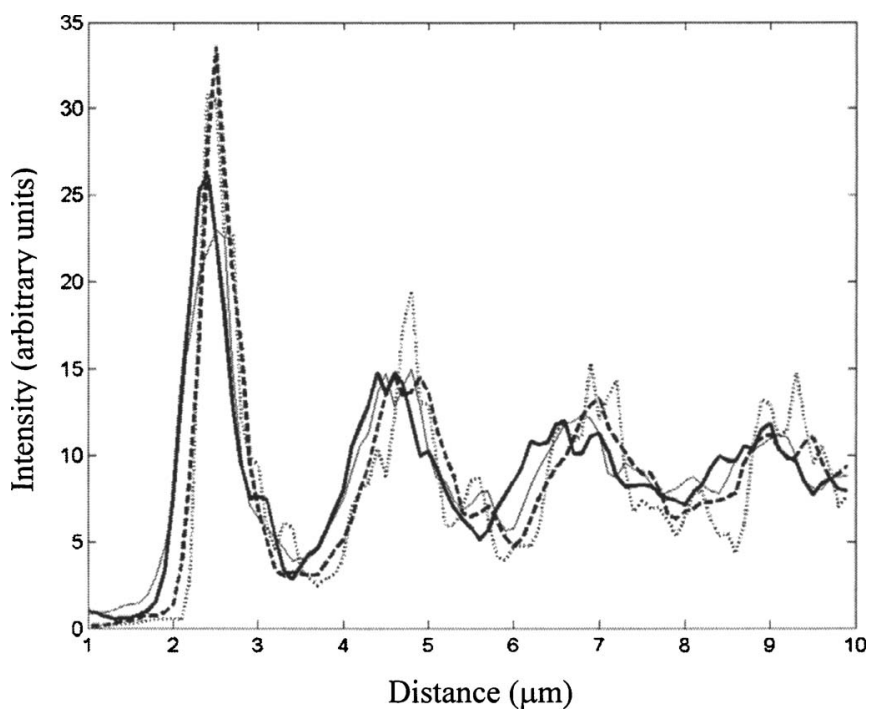

FIG. 4. Radial distribution function for sample A ( $0 \mathrm{mg} / \mathrm{ml} \mathrm{PS}$, solid line), sample B $(0.22 \mathrm{mg} / \mathrm{ml} \mathrm{PS}$, bold solid line $)$, sample C $(0.44 \mathrm{mg} / \mathrm{ml}$, dashed line $)$, and sample D $(0.88 \mathrm{mg} / \mathrm{ml}$, dotted line $)$.

scopic evidence of crystallization in this sample. While there is much structure in the $g(r)$ of sample D, the position of the first peak in that sample is not at a lower value than is that that of the other samples. Only the $g(r)$ of sample B $(0.22 \mathrm{mg} / \mathrm{ml} \mathrm{PS})$ has a first peak that occurs at a noticeably shorter distance than that of the polymer free sample A. While the position of the first peak of the $g(r)$ in sample B could indicate compaction as a result of the increased attraction relative to the nearly hard-sphere colloidal glass, this is not supported by the data for the higher polymer concentration samples, where the first peaks shift back to longer displacements. The fact that the $g(r)$ is averaged over the entire sample contributes to the difficulty in deriving details about particle spacing from this observable. Furthermore, there exists significant spatial heterogeneity in these samples and this contributes to the noise in these $g(r)$ 's compared, for example, to a $g(r)$ derived from a crystal with the same number of averaged particle positions. In sum, aside from increased structure in sample D, we find no obvious differences between the structure as reflected by the $g(r)$ of systems with nearly hard-sphere and short range attractive interactions of a variety of strengths.

\section{Ensemble averaged measures of dynamics}

The mean square displacements (MSDs) for samples A-D are plotted in Fig. 5. The MSD is plotted against Brownian time, $t D_{0} / a^{2}$, with $a$ the particle radius and $D_{0}$ the bare diffusion constant or the diffusion constant of a single colloidal particle in the same solvent-polymer solution used in the experiment. Plotting the MSDs as a function of Brownian time as opposed to real time accounts for the slower dynamics due to the increased viscosity of the background solvent in samples with increasing amounts of polymer. To determine the bare diffusion constants, the viscosity of colloid-free polymer solutions at concentrations corresponding to the free polymer concentration in our samples was measured and the Stokes-Einstein relation was used to 


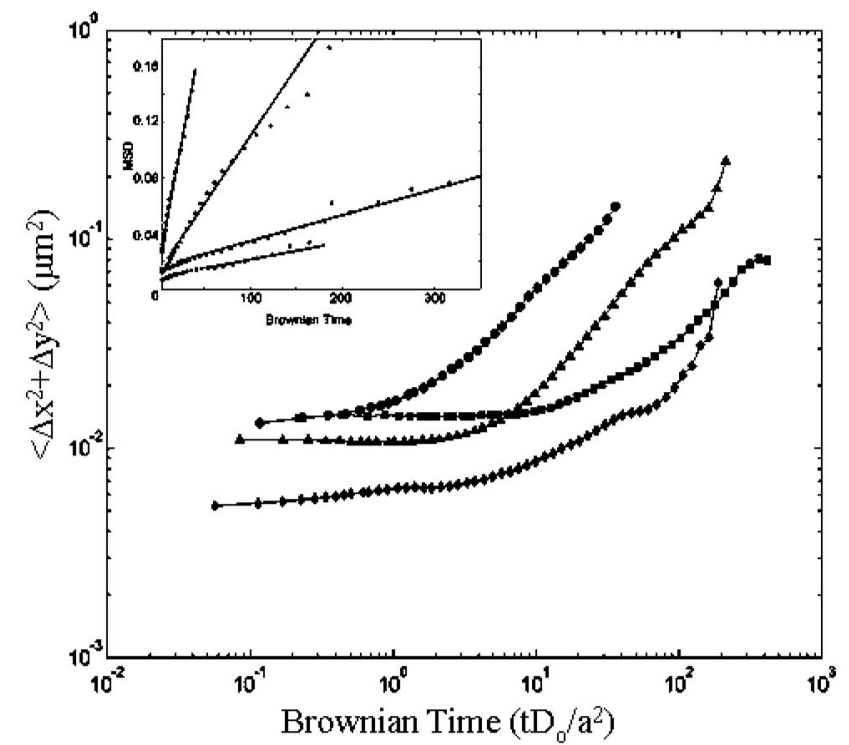

FIG. 5. MSD for sample A $(0 \mathrm{mg} / \mathrm{ml}$ PS, - -$)$, sample B $(0.22 \mathrm{mg} / \mathrm{ml}$ PS, -- -$)$, sample C $(0.44 \mathrm{mg} / \mathrm{ml} \mathrm{PS,} \mathrm{- \Lambda}-)$, and sample D $(0.88 \mathrm{mg} / \mathrm{ml}$ PS, - -). The MSD for sample B is only for the first quarter of the full experiment. Inset: linear fits of data in the diffusive regime. The slopes of these lines are used to calculate long time diffusion constants.

determine $D_{0}$ of each sample. The free polymer concentration for our samples ranges from 0 for sample A to $2.85 \mathrm{mg} / \mathrm{ml}$ for sample $\mathrm{D}$, and $D_{0}$ is $0.081,0.043,0.031$, and $0.021 \mathrm{\mu m}^{2} / \mathrm{s}$ for samples $\mathrm{A}, \mathrm{B}, \mathrm{C}$, and $\mathrm{D}$, respectively. The MSDs for all but sample B include data for runs of more than $10^{4} \mathrm{~s}$. In samples A, C, and D, the MSDs for shorter portions of the run are found to overlap those for the entire run. Thus, in these samples there does not appear to be significant changes in the dynamical behavior of the colloidal particles (as may happen due to aging, for example) during the experiment. This is not the case for sample B. In sample $\mathrm{B}$, MSDs for the first, second, third, and fourth quarters of the experiment are different, indicative of dynamics that change (and in this case, slow) on the time scale of the experiment. This result was confirmed with the (reinitiated) $0.22 \mathrm{mg} / \mathrm{ml}$ sample and with two other $0.22 \mathrm{mg} / \mathrm{ml}$ PS samples. Because of the gross dynamical changes occurring during the experiment, the MSD for sample B presented in Fig. 5 is only for the first quarter of the time run. The MSD for the entire run of sample B looks very similar to that of sample C plotted in Fig. 5.

For all samples, the MSD exhibits a plateau at early times and a subsequent upturn. The plateaus for samples A-C fall in the range of $0.011-0.014 \mu \mathrm{m}^{2}$, which corresponds to a cage of radius of $2.55 \mu \mathrm{m}$, which is $4 \%$ larger than the particle. The plateau in the MSD of sample D is noticeably lower, at $0.006 \mu \mathrm{m}^{2}$, indicating that the cages in this sample are tighter than those in the other samples. The upturn in the MSD occurs at about 1 Brownian unit in sample B, 10 in sample C, and 100 in samples A and D. In all cases, the MSD is linear for the longest times plotted (Fig. 5, inset), which allows derivation of a long time diffusion constant $D$. These values are $2.59 \times 10^{-6}, 2.75 \times 10^{-5}, 5.1$ $\times 10^{-6}$, and $7.2 \times 10^{-7} \mu \mathrm{m}^{2} / \mathrm{s}$ for samples A, B, C, and D, respectively. The value for the entire run for sample $\mathrm{B}$ is

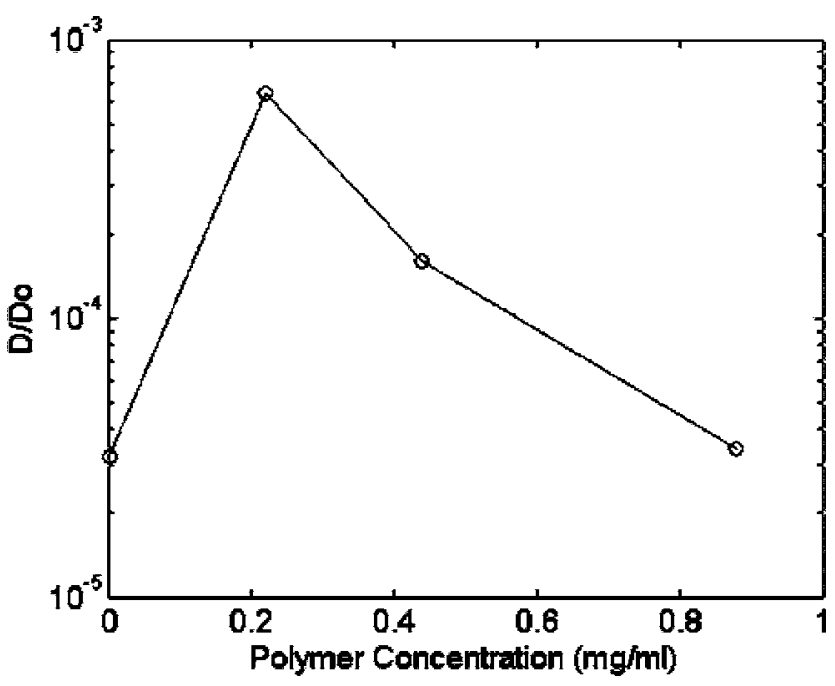

FIG. 6. Long time diffusion constant divided by bare diffusion constant as a function of sample polymer concentration.

$4.4 \times 10^{-6} \mu \mathrm{m}^{2} / \mathrm{s}$. The long time diffusion constants divided by the bare diffusion constants are shown in Fig. 6 .

The MSDs plotted in Fig. 5 and the resulting $D / D_{0}$ values plotted in Fig. 6 provide direct microscopic evidence for the reentrant glass transition first predicted by Dawson et al. ${ }^{33}$ and seen in experiment by Pham et al. ${ }^{29,31}$ and Eckert and Bartsch. ${ }^{30}$ We find two important differences between our results and those derived from the DLS measurements of Pham et al. First, we find that sample B, in the reentrant region according to our adaptation of the phase diagram of Pham et al. (Fig. 1), is clearly experiencing changes in dynamics during the experiment and shows the most liquidlike behavior at early times. Thus, the long time diffusion constant associated with the first hour of the experiment (the third hour after initiation of the sample) is noticeably faster than those derived from subsequent hours. The diffusion constant associated with the first hour of the experiment is more than an order of magnitude faster than would be expected based on the background viscosity and the volume fraction of the sample. Indeed, were there no effect from the polymer aside from increased viscosity in the sample, the MSDs in Fig. 5 would overlay each other. The fact that sample B has a MSD that has only a shallow plateau and a long time diffusion constant that is an order of magnitude faster than that of samples A and D shows that there is reentrant behavior. Upon including the entire trajectory of the experiment for sample B, an increase in the diffusion constant is still present, but it is reduced to slightly less than one order of magnitude. It is unclear what gives rise to the changing dynamics in sample B though we believe both the rapidly slowing dynamics and the fast particle rearrangements in the sample at early times in the experiment may be related to crystal nucleation and growth; we do find small crystalline areas in this sample. We have examined the order orientational parameter $Q 6$ for our samples. Here, $\quad Q 6(i)=\left[(4 \pi / 13) \sum_{m=-6}^{m=6}\left|\hat{q}_{6 m}(i)\right|^{2}\right]^{1 / 2} \quad$ and $\quad \hat{q}_{l m}(i)$ $=1 / N_{b}(i) \sum_{j=1}^{N_{b}(i)} Y_{l m}\left(\mathbf{r}_{i j}\right)$ with $N_{b}(i)$ the number of neighbors of the particle, $Y_{l m}$ a spherical harmonic, and $r_{i j}=r_{j}-r_{i}$ with $r_{i}$ the coordinates of the tagged particle. ${ }^{55}$ Though we find clear 
differences between 2D slices of 3D crystals and 2D slices of 3D glasses, we see no obvious differences between glassy samples in the repulsive, reentrant, and attractive regions of the phase diagram (not shown). We believe this is because only $\sim 5 \%$ of the particles in samples of composition B are present in these small crystalline areas. More support for the idea that we are seeing the start of crystallization in sample B comes from the fact that Pham et al. find samples in this regime to crystallize within one day in contrast to the repulsive and attractive glasses, which do not crystallize within four weeks. ${ }^{31}$ Furthermore, visual observation of early time rearrangements in sample B shows these dynamics resemble the dynamics found in samples with $\phi=0.58$ and 0.22 and $0.44 \mathrm{mg} / \mathrm{ml}$ PS. These samples crystallize macroscopically within 6 hs when loaded in microscopy sample cells, and visualization of this process shows considerable motion of the particles as small crystalline areas grow. The motion tends to occur in extended, or stringlike, regions, much as they do in sample B.

A second difference between our results and those of Pham et al. is that sample $\mathrm{C}$, which falls in the attractive glass region of our adaptation of the phase diagram of Pham et al., displays dynamics more similar to those of sample B than to those of sample D. Sample C behaves more like a reentrant sample than a glassy one, though it does not display the obvious changes in dynamical behavior seen in sample B. We believe that sample $\mathrm{C}$ is very close to the line between the reentrant and attractive glass regions. This suggests that the line defining the attractive glass in our system is at a deeper attractive well depth at $\phi=0.60$ than in the system of Pham et al. Our experiment differs from the experiments of Pham et al. in several ways that may account for this difference. First, our experiments are done with the salt TBAC, which should screen any surface charges on the particles, while the experiments of Pham et al. were not. If there are surface charges on the particles (the chances of this are lower in the experiments of Pham et al. than here since those experiments employ decalin, rather than decalin and $\mathrm{CXB}$, as solvent), the effective particle size is larger than the actual size of the particles. Thus, the effective volume fraction is higher than it would be in a system with any surface charges screened. In the case that the particles in the system of Pham et al. are slightly charged relative to those here, the movement of the attractive glass line seems plausible. We can also not rule out that particle-specific differences (preparation, polydispersity, size, and volume fraction relative to RCP) may contribute to the measured differences. Furthermore, differences in solvent and sedimentation rate may affect the conclusions. We aim to eliminate sedimentation in our samples by using a nearly density-matched solvent. However, in the work of Pham et al. sedimentation is less of a concern because of the small size of the particle, and as a result no attempt is made to density match the particle and background solvent.

A further difference between the results presented here and those in Refs. 29 and 31 is how appreciably the diffusion constant speeds up in the reentrant region. In our experiments, sample B has a diffusion constant that is 20 times faster than would be expected based on the volume fraction and background viscosity (Fig. 6). While long time diffusion constants are not reported in the work of Pham et al., the intermediate scattering functions at a wavelength corresponding to approximately four particle diameters suggest the relaxation of the samples in the reentrant region is approximately three orders of magnitude faster than in the glassy samples. The difference in relaxation times varies with wave vector, and at the peak of the structure factor the differences are even more pronounced. While our results do not show such drastic differences, our results are consistent with recent simulations. ${ }^{56}$ In the work of Reichman et al., it is found that simulated high density systems with very weak and strong attractions have similar long time diffusion constants while those with attractions of intermediate strength (the reentrant samples) have diffusion constants that are a maximum of two orders of magnitude faster than those with very weak and strong attractions. At slightly higher and lower polymer concentrations than that which gives rise to the fastest diffusion constant measured, the diffusion constants are one order of magnitude faster than those of the systems with very weak and strong attractions. This is consistent with our results.

The upturn often seen in mean square displacements of glassy systems has been associated with cage escape. The non-Gaussian parameter for measurements in $2 \mathrm{D}$ is $\alpha_{2}(t)=\frac{1}{2}\left\langle\delta r^{4}\right\rangle /\left\langle\delta r^{2}\right\rangle^{2}-1$, with $\delta r$ the distance a particle moves in time $t$. This quantity generally peaks at a time similar to that of the MSD upturn. The peak in the nonGaussian parameter then also corresponds to the time at which an average particle escapes its cage. The peak of the non-Gaussian parameter has also been shown to correspond approximately to the time at which the dynamic heterogeneity in the sample is a maximum. We find peaks in the nonGaussian parameter [Fig. 7(a)] at $\sim 30,2$, and 9 in Brownian units for samples A and B (the entire run) and C, respectively. No clear peak is apparent in the non-Gaussian parameter in sample D. The peak positions in the non-Gaussian parameter track the upturn in the MSDs and the diffusion constants derived from the MSDs as expected, again in agreement with the findings of Reichman et al. ${ }^{56}$ The amplitude of the non-Gaussian parameter peaks at 4 for both samples A and B and at 5 for sample C. Had data been collected long enough to see a peak in the non-Gaussian parameter in sample D, we might expect it to have a larger non-Gaussian parameter, as is seen in the most highly attractive systems studied in Ref. 56.

Recently, an alternative non-Gaussian parameter was proposed by Flenner and Szamel. ${ }^{57}$ This alternative nonGaussian parameter for 2D measurements of a 3D system is $\gamma(t)=\frac{1}{2}\left\langle\delta r^{2}\right\rangle\left\langle 1 / \delta r^{2}\right\rangle-1$ and weights small displacements as heavily as large ones and thus will peak at the time at which the relaxation is most heterogeneous, which is expected to be longer than the time at which the traditional non-Gaussian parameter, $\alpha_{2}(t)$, peaks. Figure 7(b) displays the alternative non-Gaussian parameters for the four samples studied versus Brownian time. In all cases, the shape is similar to that predicted for glassy systems, though in all cases data have not been collected for long enough times to show a clear peak. There are indications of a peak in sample $\mathrm{C}$ at $\sim 30$ units in 

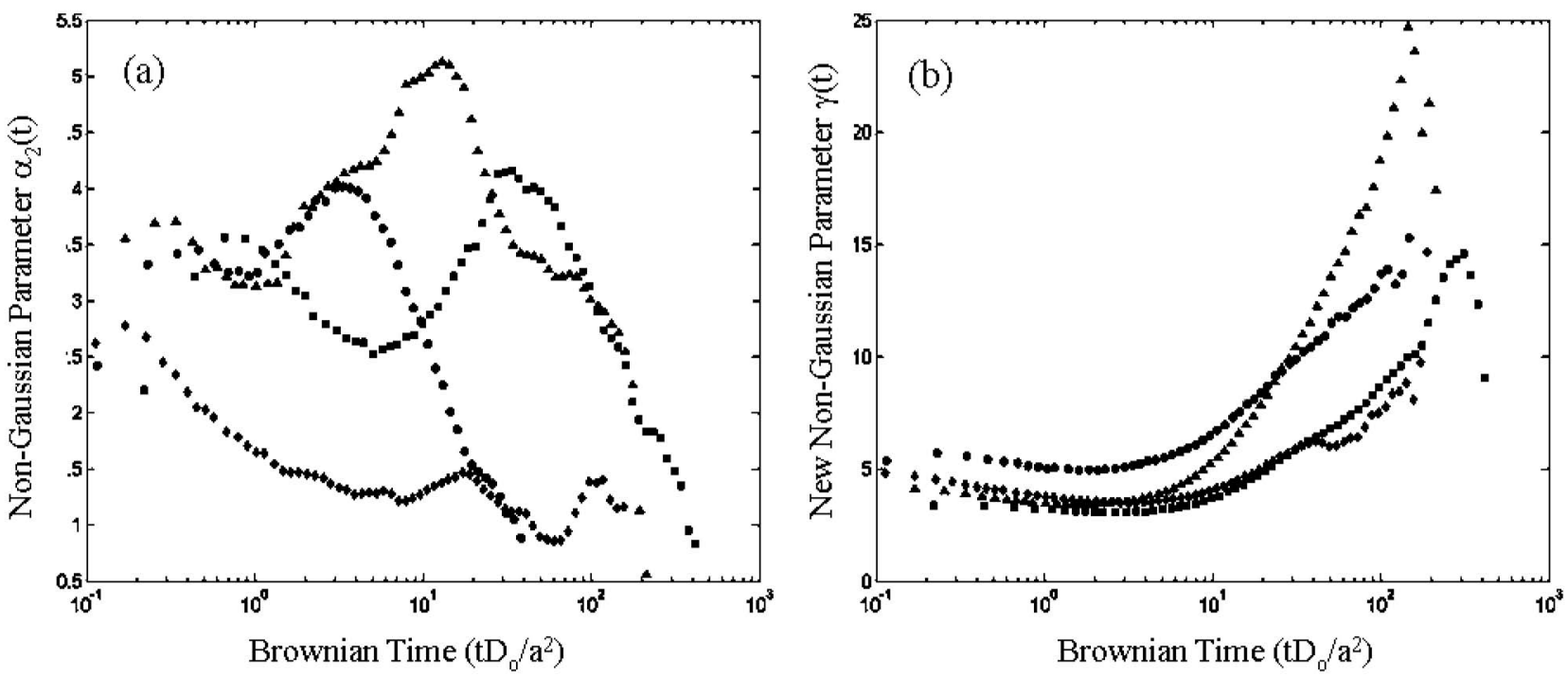

FIG. 7. (a) Traditional non-Gaussian parameter for sample A ( $0 \mathrm{mg} / \mathrm{ml} \mathrm{PS,} \mathrm{- \square -),} \mathrm{sample} \mathrm{B}(0.22 \mathrm{mg} / \mathrm{ml} \mathrm{PS},-\mathbf{-})$, sample C (0.44 mg/ml PS, - $\mathbf{\Delta}$-), and sample D $(0.88 \mathrm{mg} / \mathrm{ml}$ PS, - -). (b) Alternative non-Gaussian parameter for samples A-D with same symbols as in panel (a).

Brownian time and at $\sim 70$ units in Brownian time for sample A. Longer time traces would be useful in seeing whether the amplitude of this alternative non-Gaussian parameter confirms what is seen in the images collected, that attractive glasses display greater dynamic heterogeneity than systems in either the repulsive glass or reentrant regions.

\section{Individual and few particle dynamics}

While the ensemble averaged observables measured are largely consistent with those measured by DLS, microscopic imaging allows direct examination of the microscopic basis for the differences between the ensemble averaged quantities measured in repulsive and attractive glasses. When visualizing the repulsive glasses, reentrant samples, and attractive glasses with CARS microscopy we find strikingly different dynamics between the three types of systems. Repulsive glasses exhibit cage rattling: the particles move back and forth within cages defined by their nearest neighbors. ${ }^{21,22,44}$ Plotting trajectories of all the particles over time also provides clear evidence for dynamic heterogeneity, as has been seen by others visualizing motion in hard-sphere colloidal glasses. $^{21,22,44}$ However, unlike some previous findings, we find that the most mobile regions tend to be compact as opposed to stringlike or extended. Notably, in no samples with polymer is cage rattling seen. In sample B there is motion that appears almost free (or diffusive) at early times [as confirmed by the short plateau in the MSD (Fig. 5)]. Sometimes this motion precedes crystallization in small areas of the plane being imaged and sometimes it precedes a glassy state exhibiting very little motion at long times. Like sample $\mathrm{B}$, sample $\mathrm{C}$ displays significant motion, but never the free motion seen in sample $\mathrm{B}$. The motion in sample $\mathrm{C}$ is similar in character to that in sample $\mathrm{D}$, but there is noticeably more motion in sample C. In both samples C and D the particle motion is saltatory and abrupt. In sample D most particles move very little on the time scale of the experiment. However, when a particle moves, it does so quickly and over an appreciable distance. In a repulsive glass, such as sample A, no motion on the order of more than a $\frac{1}{4}$ particle diameter is ever seen between consecutive frames $(3.9 \mathrm{~s})$. In an attractive glass, such as sample D, however, a given particle may move on the order of a particle diameter in the same time interval. The upper panel of Fig. 8 shows typical consecutive images (3.9 s apart) of the polymer-free repulsive glass, sample A. The rightmost image is a difference image and shows that in one area of the field of view there is enough rearrangement of particles between the images to give rise to intensity modulations in the difference image. The lower panel of Fig. 8 shows an example of particle motion in the attractive glass, sample D. While the difference image shows no visible modulation over the bulk of the area imaged, there is one location that shows marked motion of several particles, including one that moves almost a particle diameter in $3.9 \mathrm{~s}$. Though we believe this to be a particle moving in the $x y$ plane, there is also the possibility that one particle moves out of the focal plane while another one moves into it. Such motion would be similarly unusual, as it too would require particles to move a full particle diameter in less than $4 \mathrm{~s}$. Indeed, the fact that the difference image in the lower panel of Fig. 8 does not have equally sized regions that are both darker and lighter than the background indicates that some of the motion occurs outside of the 2D slice imaged, as is expected in a 3D sample. While motions such as those depicted in the lower panel of Fig. 8 do not occur frequently in the attractive glass, they do occur, whereas these types of motions are never seen in either sample A or B. Movies of this type of motion underscore that the dynamics in the attractive glass are not well described by cage rattling and cage escape and that attractive glasses have highly heterogeneous dynamics, as seen previously in simulation. ${ }^{36,56}$ Figure 8 also reinforces that, while the long time diffusion constant as measured by the MSD is similar in samples A and D, the microscopic dynamics that give rise to these diffusion constants are vastly different. The types of motion seen in the repulsive and attractive glasses and displayed in Fig. 8 are 

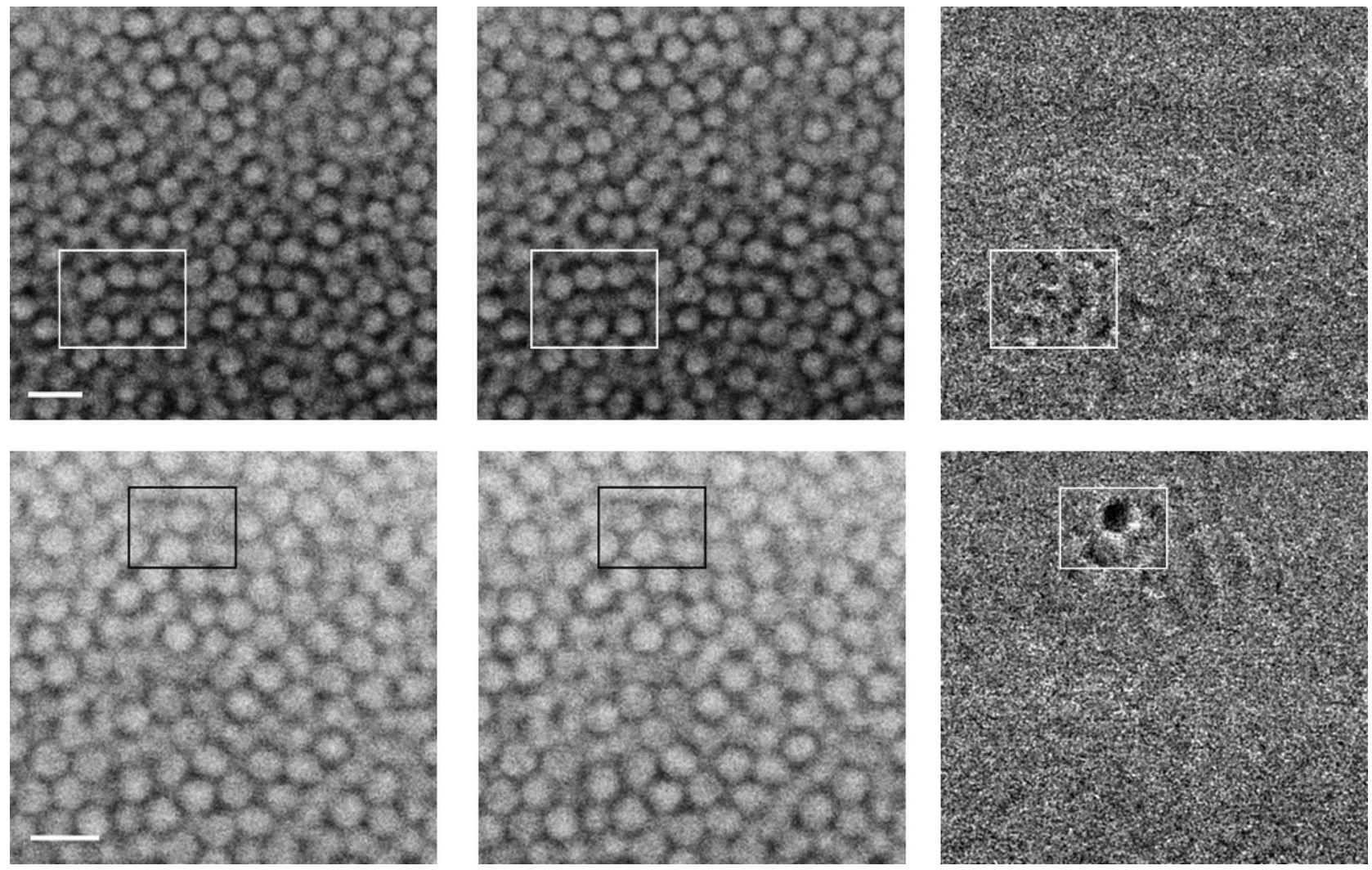

FIG. 8. Panels from left to right: sample at time $t$, sample at time $t+3.9 \mathrm{~s}$, and difference image. Upper panels: typical motion in a hard sphere glass (sample A, $0 \mathrm{mg} / \mathrm{ml} \mathrm{PS})$. Lower panels: motion seen in sample D $(0.88 \mathrm{mg} / \mathrm{ml}$ PS $)$. Contrast is the same in upper and lower panel difference images. Scale bar is $5 \mu \mathrm{m}$.

consistent with the trajectories found in Ref. 56 for samples with low and high polymer concentrations. ${ }^{56}$ In these simulations, the 5\% least mobile particles in the weakly attractive system move significantly further (i.e., they rattle in a large cage) than do those with a strong attractive interaction, which appear almost static. Conversely, the 5\% most mobile particles in the weakly attractive system exhibit displacements that are much smaller than the 5\% most mobile particles in the highly attractive system. Thus, the differential in average motion between the least and most mobile particles is much greater in the attractive glass than in the repulsive glass, as is seen in the CARS images.

Though we find different types and degrees of heterogeneity in samples in the repulsive glass, reentrant, and attractive glass regions, in all cases except at early times in sample $\mathrm{B}$, we find compact rather than extended areas of fast particles. In samples at $\phi=0.58$, extended areas of increased motion in both samples with and without polymer are present. However, in these samples as well as at early times in sample B, stringlike motion occurs, most noticeably adjacent to small crystalline regions. We believe that the cooperative extended areas of mobility in these regions is associated with the growth of a nucleated crystal.

In addition to the samples discussed thus far, images of a sample with $10 \mathrm{mg} / \mathrm{ml} \mathrm{PS}$, well above the overlap concentration, were collected. As mentioned previously, this sample shows obvious structural differences from all other samples studied. In this sample, we find dynamics similar to that in sample D, but with more obvious cooperative motion. Figure
9 depicts one instance of notable cooperative motion. In the sequence of images before those depicted in Fig. 9, there is no obvious motion for considerable time. Then, one particle, that with the longest open headed arrow in Fig. 9, makes a sudden jump of close to a particle diameter in less than $4 \mathrm{~s}$. In the subsequent frames, a cluster of particles in the plane undergoes a cooperative, clockwise rotational motion. In this case it appears that one particle breaks free from its cage and moves in a way that results in a high free energy state. However, the particles that would normally move individually to

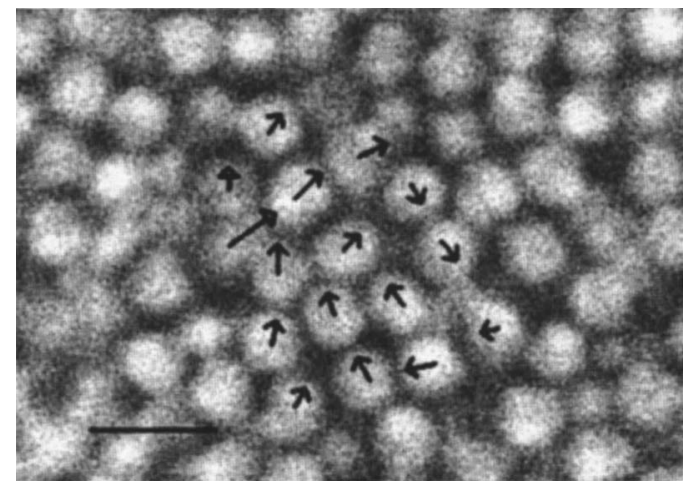

FIG. 9. Cooperative motion seen in $10 \mathrm{mg} / \mathrm{ml}$ PS sample. Arrows indicate direction and magnitude of motion of a cluster of highly mobile particles in the sample. The arrow length is twice the actual colloid displacement. The two arrows with open heads indicate particles that moved significantly out of the imaged plane during the time interval discussed. Image is taken at time $t, 1121 \mathrm{~s}$ into the experiment. Arrows indicate the motion between time $t$ and a time $22 \mathrm{~s}$ later. Scale bar is $5 \mu \mathrm{m}$. 
accommodate this particle are coupled by attractive forces strong enough to require the particles to move as a unit. The cooperative motion seen in this sample appears to be a more extreme version of the motion visualized in sample $\mathrm{D}$, in which short bursts of motion are followed by cooperative rearrangements of nearby particles.

The noticeably different dynamics of systems in the repulsive glass, reentrant, and attractive glass regions as seen in samples A-D can be understood by considering that the cages in systems with polymer will be tighter than those in systems with nearly hard-sphere interactions. These cages appear to prevent rattling: this is due to a combination of increased local volume fraction within the cage and the attractions (or in the case of very strong attractions, bonds) between the particles. Since we only see obviously cooperative, simultaneous particle motion characteristic of affixed particles in the $10 \mathrm{mg} / \mathrm{ml}$ PS sample, we believe very strong attractions are necessary for this type of motion. However, even without such strong attractions, we see clear differences between the dynamics in samples A-D, which is due at least in part to the increased local volume fraction of cages as a function of polymer concentration. If the cages in samples with polymer do indeed have a higher volume fraction, then in all such systems, both those in the reentrant and attractive glass regimes, there must be areas with lower volume fraction than that of the tight cages. In the reentrant region, these areas of lower volume fraction presumably allow for easier rearrangement once a particle is free from the tight cage. This leads to the faster relaxation of the system, which pushes the glass transition to higher volume fractions than in nearly hard-sphere systems. In attractive glasses, though there must be areas of relatively low local volume fraction, here the attractive interactions between a particle and its neighbors are strong enough to prevent almost all cage escape. This results in the very slow relaxation of the attractive glasses. This interpretation is consistent with the DLS experiments on attractive glasses, in which the intermediate scattering functions lack the characteristic plateau associated with motion within a cage and decay associated with cage escape. Instead, the intermediate scattering functions measured in those experiments and, to a lesser extent, the MSD measured in the experiments presented here indicate that the attractive glass has a very slow decay that accelerates at long times. These results confirm that the motion in attractive glasses is not well described by rattling within a cage followed by escape from that cage, presumably because the cage is tight and strongly held together. Since the attractions between particles in the cage are strong, it is unlikely that multiple particles within the cage move simultaneously in a way that opens up a void that allows for particle escape. When this type of fluctuation does occur, strong attractions make cooperative rearrangement likely.

\section{CONCLUSION}

CARS microscopy has been used to study the microscopic structure and dynamics of a range of colloidal samples at high density, from those with no polymer and a nearly hard-sphere interaction to those with a strong attrac- tive interaction. Intermediate polymer samples are seen to have faster, less heterogeneous dynamics than either repulsive or strongly attractive glasses. These observations are in accord with previous DLS experiments, though we do not find agreement with the precise position of the reentrant glass transition line determined in those experiments.

In the experiments presented here, most insight is gained via direct observation of the images rather than via ensemble averaged quantities. It is found that repulsive and attractive glasses have qualitatively different dynamics. While the repulsive glasses are well described by cage rattling and escape, attractive glasses are not. Instead, the particles comprising an attractive glass usually move very little, but occasionally make large jumps in a short time, exhibiting saltatory and cooperative motion.

Microscopic experiments continue to reveal details of the dynamics in colloidal samples that are not accessible with ensemble averaged experiments. In the case of repulsive versus attractive glasses, the dynamics and dynamic heterogeneities in each have been shown to be clearly different. It will be interesting to microscopically explore glasses with intermediate polymer concentrations at volume fractions higher than $\phi=0.60$. These experiments would test for the presence of the A 3 singularity, where the distinction between the repulsive and attractive glasses disappears.

\section{ACKNOWLEDGMENTS}

We thank J. Conrad, S. Manley, and H. Wyss for assistance in light scattering and rheology measurements as well as for useful discussions. We also thank P. Lu and D. Reichman for helpful discussions. We thank A. Schofield for supplying particles. Finally, we thank X. S. Xie and CIMS for the use of equipment.

${ }^{1}$ P. N. Pusey and W. Van Megen, Nature (London) 320, 340 (1986).

${ }^{2}$ P. N. Pusey and W. Van Megen, Phys. Rev. Lett. 59, 2083 (1987).

${ }^{3}$ P. N. Segre, S. P. Meeker, P. N. Pusey, and W. C. K. Poon, Phys. Rev. Lett. 75, 958 (1995).

${ }^{4}$ G. Bryant, S. R. Williams, L. Qian, I. K. Snook, E. Perez, and F. Pincet, Phys. Rev. E 66, 060501 (2002).

${ }^{5}$ W. G. Hoover and F. H. Ree, J. Chem. Phys. 49, 3609 (1968).

${ }^{6}$ L. V. Woodcock and C. A. Angell, Phys. Rev. Lett. 47, 1129 (1981).

${ }^{7}$ W. Schaertl and H. Sillescu, J. Stat. Phys. 74, 687 (1994).

${ }^{8}$ W. Schaertl and H. Sillescu, J. Stat. Phys. 77, 1007 (1994).

${ }^{9}$ B. Doliwa and A. Heuer, Phys. Rev. Lett. 80, 4915 (1998).

${ }^{10}$ W. Van Megen and P. N. Pusey, Phys. Rev. A 43, 5429 (1991).

${ }^{11}$ G. D. Scott and D. M. Kilgour, Br. J. Appl. Phys. 2, 863 (1969).

${ }^{12}$ S. Torquato, T. M. Truskett, and P. G. Debenedetti, Phys. Rev. Lett. 84, 2064 (2000).

${ }^{13}$ W. Van Megen and S. M. Underwood, Phys. Rev. E 47, 248 (1993).

${ }^{14}$ W. Van Megen and S. M. Underwood, Phys. Rev. E 49, 4206 (1994).

${ }^{15}$ T. G. Mason and D. A. Weitz, Phys. Rev. Lett. 75, 2770 (1995).

${ }^{16}$ R. Bohmer, K. L. Ngai, C. A. Angell, and D. J. Plazek, J. Chem. Phys. 99, 4201 (1993).

${ }^{17}$ L. A. Deschenes and D. A. Vanden Bout, Science 292, 255 (2001).

${ }^{18}$ G. Adam and J. H. Gibbs, J. Chem. Phys. 43, 139 (1965).

${ }^{19}$ A. H. Marcus, J. Schofield, and S. A. Rice, Phys. Rev. E 60, 5725 (1999).

${ }^{20}$ W. K. Kegel and A. van Blaaderen, Science 287, 290 (2000).

${ }^{21}$ E. R. Weeks, J. C. Crocker, A. C. Levitt, A. Schofield, and D. A. Weitz, Science 287, 627 (2000).

${ }^{22}$ E. R. Weeks and D. A. Weitz, Phys. Rev. Lett. 89, 095704 (2002).

${ }^{23}$ E. R. Weeks and D. A. Weitz, Chem. Phys. 284, 361 (2002).

${ }^{24}$ H. M. Lindsay and P. M. Chaikin, J. Chem. Phys. 76, 3774 (1982).

${ }^{25}$ A. Yethiraj and A. van Blaaderen, Nature (London) 421, 513 (2003). 
${ }^{26}$ H. N. W. Lekkerkerker, W. C. K. Poon, P. N. Pusey, A. Stroobants, and P. B. Warren, Europhys. Lett. 20, 559 (1992).

${ }^{27}$ W. C. K. Poon, J. S. Selfe, M. B. Robertson, S. M. Ilett, A. D. Pirie, and P. N. Pusey, J. Phys. II 3, 1075 (1993).

${ }^{28}$ F. Mallamace, P. Gambadauro, N. Micali, P. Tartaglia, C. Liao, and S. H. Chen, Phys. Rev. Lett. 84, 5431 (2000).

${ }^{29}$ K. N. Pham, A. M. Puertas, J. Bergenholtz, S. U. Egelhaaf, A. Moussaid, P. N. Pusey, A. B. Schofield, M. E. Cates, M. Fuchs, and W. C. K. Poon, Science 296, 104 (2002).

${ }^{30}$ T. Eckert and E. Bartsch, Faraday Discuss. 123, 51 (2003).

${ }^{31}$ K. N. Pham, S. U. Egelhaaf, P. N. Pusey, and W. C. K. Poon, Phys. Rev. E 69, 011503 (2004).

${ }^{32}$ J. Bergenholtz and M. Fuchs, Phys. Rev. E 59, 5706 (1999).

${ }^{33}$ K. Dawson, G. Foffi, M. Fuchs, W. Gotze, F. Sciortino, M. Sperl, P. Tartaglia, Th. Voigtmann, and E. Zaccarelli, Phys. Rev. E 63, 01140 (2000).

${ }^{34}$ F. Sciortino, P. Tartaglia, and E. Zaccarelli, Phys. Rev. Lett. 91, 268301 (2003).

${ }^{35}$ A. M. Puertas, M. Fuchs, and M. E. Cates, Phys. Rev. Lett. 88, 098301 (2002).

${ }^{36}$ A. M. Puertas, M. Fuchs, and M. E. Cates, J. Chem. Phys. 121, 2813 (2004).

${ }^{37}$ S. E. Phan, W. B. Russel, J. X. Zhu, and P. M. Chaikin, J. Chem. Phys. 108, 9789 (1998).

${ }^{38}$ M. D. Duncan, J. Reintjes, and T. J. Manuccia, Opt. Lett. 7, 350 (1982).

${ }^{39}$ J. X. Cheng, A. Volkmer, and X. S. Xie, J. Opt. Soc. Am. B 19, 1363 (2002).
${ }^{40}$ J. X. Cheng, Y. K. Jia, G. F. Zheng, and X. S. Xie, Biophys. J. 83, 502 (2002).

${ }^{41}$ J. X. Cheng and X. S. Xie, J. Phys. Chem. B 108, 827 (2004).

${ }^{42}$ J. C. Crocker and D. G. Grier, J. Colloid Interface Sci. 179, 298 (1996).

${ }^{43}$ A. D. Dinsmore, E. R. Weeks, V. Prasad, A. C. Levitt, and D. A. Weitz, Appl. Opt. 40, 4152 (2001).

${ }^{44}$ P. Habdas and E. R. Weeks, Curr. Opin. Colloid Interface Sci. 7, 196 (2002).

${ }^{45}$ S. Asakura and F. Oosawa, J. Polym. Sci. 33, 183 (1958).

${ }^{46}$ S. Asakura and F. Oosawa, J. Chem. Phys. 22, 1255 (1954).

${ }^{47}$ A. Vrij, Pure Appl. Chem. 48, 471 (1976).

${ }^{48}$ S. M. Ilett, A. Orrock, W. C. K. Poon, and P. N. Pusey, Phys. Rev. E 51, 1344 (1995).

${ }^{49}$ H. N. W. Lekkerkerker, Colloids Surf. 51, 419 (1990).

${ }^{50}$ E. J. Meijer and D. Frenkel, J. Chem. Phys. 100, 6873 (1994).

${ }^{51}$ L. J. Fetters, N. Hadjichristidis, J. S. Lindner, and J. W. Mays, J. Phys. Chem. Ref. Data 23, 619 (1994)

${ }^{52}$ K. Terao and J. W. Mays, Eur. Polym. J. 40, 1623 (2004).

${ }^{53}$ T. Ohta and Y. Oono, Phys. Lett. 89A, 460 (1982).

${ }^{54}$ L. Fabbian, W. Gotze, F. Sciortino, P. Tartaglia, and F. Thiery, Phys. Rev. E 59, R1347 (1999).

${ }^{55}$ I. Volkov, M. Cieplak, J. Koplik, and J. R. Banavar, Phys. Rev. E 66, 061401 (2002).

${ }^{56}$ D. R. Reichman, E. Rabani, and P. L. Geissler, J. Phys. Chem. B 109, 14654 (2005)

${ }^{57}$ E. Flenner and G. Szamel, Phys. Rev. E 72, 031508 (2005). 